\title{
A novel 5-DOF high-precision compliant parallel mechanism for large-aperture grating tilling
}

\author{
Zhongxi Shao, Shilei Wu, Jinguo Wu, and Hongya Fu \\ School of Mechanical Engineering, Harbin Institute of Technology, Harbin 150001, China \\ Correspondence to: Hongya Fu (hongyafu@ hit.edu.cn)
}

Received: 27 July 2017 - Revised: 26 October 2017 - Accepted: 3 November 2017 - Published: 30 November 2017

\begin{abstract}
In combination with the advantages of parallel mechanisms and compliant mechanisms, a 5-DOF compliant parallel mechanism is designed to meet the requirements, such as large stroke, large load capacity, high precision and high stability, for a large-aperture grating tiling device. The structure and characteristics of the 5-DOF compliant parallel mechanism are presented. The kinematics of the mechanism are derived based on a pseudo-rigid-body model as well. To increase the tiling position retention stability of the mechanism, a closedloop control system with capacitive position sensors, which are employed to provide feedback signals, is realized. A position and orientation monitoring algorithm and a single neuron adaptive full closed-loop control algorithm are proposed. Performance testing is implemented to verify the accuracy and the tiling position retention stability of the grating tiling device. The experimental results indicate that the tiling accuracy reaches $0.2 \mu \mathrm{rad}$ per step and $20 \mathrm{~nm}$ per step, and the tiling position retention stability can achieve $1.2 \mu \mathrm{rad}$ per $30 \mathrm{~min}$ and $35 \mathrm{~nm}$ per $30 \mathrm{~min}$ in the rotational direction and the translational direction, respectively.
\end{abstract}

\section{Introduction}

Large-aperture diffraction grating is an essential element applied in many high-tech research fields, such as precision measuring instruments, optical communications and inertial confinement fusion. The currently available singlediffraction grating with a size less than $1 \mathrm{~m}$ cannot meet the large-aperture requirements, so it is critical to solve the problem of large-aperture diffraction grating. The tiling technique, which tiles multiple small-scale grating segments into one large grating, can be used to deal with this problem. The tiling orientation and position of two adjacent small-scale gratings should have high precision, and this precision should be maintained for a long time as well. Thus, it is important to investigate an ultra-precision positioning mechanism of high precision and high stability.

In order to achieve such high precision and stability, grating tiling theoretical analysis and structure design have respectively been studied in past decades (Bai et al., 2013; Yang et al., 2012). Zhang et al. (1998) derived the theoretical models by including various angular errors for the optical design of the array-grating compressor and analyzed the influence of angular errors on temporal performance. Kessler et al. (2004) proposed a pairing error compensation theory, which reduced the number of control variables to three. Only by controlling the longitudinal piston, angular tip and angular tilt can the grating tiling device be properly controlled. Rochester University designed a tetrahedral brace grating tiling device, OMEGA-EP, which achieved long-term tiling stability by using the interferometric near-field method (Qiao et al., 2007). The hierarchical grating tiling device was designed to meet the requirements of PETAL (Hornung et al., 2007). Habara et al. (2010) utilized three different dimensional capacitive position sensors to realize high-precision grating tiling for FIREX.

From the above literature review, it can be seen that the grating tiling technique has become a crucial method for achieving large-aperture grating. However, the research on grating tiling mechanism precision and stability lags behind the development of grating tiling theory. This paper focuses on the design of an ultra-precision positioning mechanism for a grating tiling system. Recently, many varieties of precision positioning mechanisms have been studied. Shi et al. (2013, 2014) and Shi and $\mathrm{Hu}$ (2013) presented a flexure-based hexapod nano-positioner with small displacement, high resolution 
and high accuracy. Yun et al. (2010) presented a novel 6DOF dual-redundant compliant parallel mechanism, and the mechanism could achieve a nanometer scale in the millimeter range. Liang et al. (2011) also presented an ultra-precision positioning based on compliant parallel mechanisms, which was integrated with a force sensor to provide force feedback. Yu et al. (2015) and Hao and Yu (2016) designed an improved large-range decoupled $X Y$ compliant parallel micromanipulator. A 6-SPS compliant positioning manipulator was proposed, which was designed based on piezoelectric actuators, flexure hinge and parallel structure by $\mathrm{Du}$ et al. (2014). A novel piezoelectric actuator 6-DOF precision positioning system, which was assembled with two 3DOF compliant parallel mechanisms, was presented in Cai et al. (2017). From the literature review, it is noted that positioning mechanisms can be considered a system of combinations of piezoelectric actuators, flexure hinges and a parallel structure. However, most of the developed positioning mechanisms lack enough lateral stiffness and loading capacity. Therefore, we aim to design a novel compliant parallel mechanism with high lateral stiffness and large load capacity. In general, the screw theory approach (Su et al., 2009), freedom and constraint topology (Hopkins and Culpepper, 2010) and the constraint-based approach (Hale, 1999) are three common methods used to design compliant parallel mechanisms. With a constraint-based approach, Hao and Kong (2016) and Hao $(2016,2017)$ presented an actuation-leg addition method by adding the same number of 6-DOF legs to an original mechanism. This method can increase the stiffness in the DOF directions, which is suitable for high lateral stiffness applications.

Based on the above advances, a novel 5-DOF micro-nano compliant parallel mechanism with three orthogonal planes and a single-point supporting structure layout based on the actuation-leg addition method is proposed in this paper. Such a micro-nano combination design strategy increases motion stroke and improves the positioning precision. The layout improves lateral stiffness and ensures that the mechanism has high stability, large load capacity and rapid response. This paper is organized as follows: Sect. 2 presents the design and characteristics of the 5-PSS/SSP-PPS compliant parallel mechanism. Section 3 provides the kinematic analysis of the mechanism. The full closed-loop control system for high stability is described in Sect. 4. Section 5 shows the experimental system and results. Finally, conclusions are drawn in Sect. 6.

\section{Grating tiling mechanism design}

\subsection{Process of grating tiling}

As is shown in Fig. 1, two adjacent gratings form a largeaperture grating. In order to make the tiling errors meet the requirements, 5-DOF adjustments should be involved between adjacent small-scale gratings: lateral shift $\Delta x$, longi-

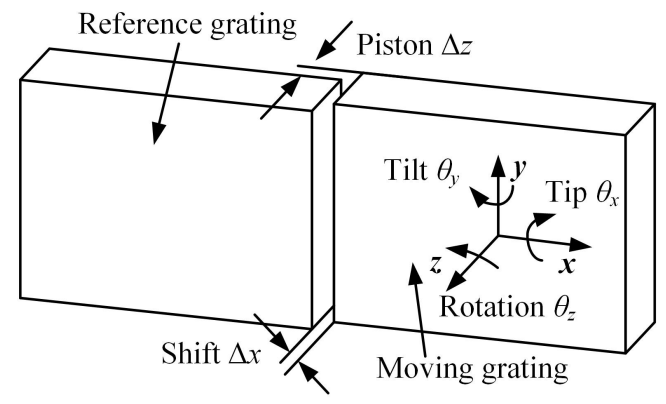

Figure 1. Schematic diagram of grating tiling.

tudinal piston $\Delta z$, angular tip $\theta_{x}$, angular tilt $\theta_{y}$ and linear rotation $\theta_{z}$. An inertial confinement fusion system requires that the tiling accuracy of translation and rotation in the millimeter range be kept on the micro-radian and nanometer scale and be maintained stably for a long time. Therefore, an ultraprecision grating tiling mechanism with large stroke, high precision and high stability should be designed.

\subsection{Design of mechanical structure}

In the design of a grating tiling device, the actuation-leg addition method is selected as the design method because it can increase the stiffness in the DOF directions. There are three steps in this design method. Firstly, design an $n$-DOF mechanism as an original mechanism. Secondly, design 6-DOF legs of the same number as the original mechanism, where each leg consists of a 1-DOF translational joint and a 5-DOF rotational joint. Finally, connect an extra leg to the motion stage of the original mechanism. The design of a 5-DOF grating tiling mechanism starts by designing an original 5-DOF mechanism. This original mechanism consists of a steel ball ( $\mathrm{S}$ joint) and two vertical installation linear guides (P joint), which play the role of supporting, guiding and positioning. By adding five extra 6-DOF legs where each leg consists of two prismatic joints $(\mathrm{P})$ and two spherical joints (S), a 5-DOF grating tiling mechanism is obtained as shown in Fig. 2, and a schematic diagram is presented in Fig. 3. An inertial confinement fusion system requires that the grating tiling mechanism achieve a nanometer scale in the millimeter range. The step motor equipped with a ball screw is characterized by large stroke and highly accurate speed and position control. But, it is difficult to achieve nano-precision adjustment. While the PZT actuators can achieve nanometer positioning accuracy, the motion range is generally very small. Hence, the step motor (macromotion) and PZT actuators (micromotion) are adopted as dual actuators to distinguish them from the conventional system, which ensures not only high precision but also large stroke. The spatial 5-PSS-PPS parallel structure is adopted as macromotion, while the micromotion is provided by a spatial 5-SSP-PPS parallel structure. 


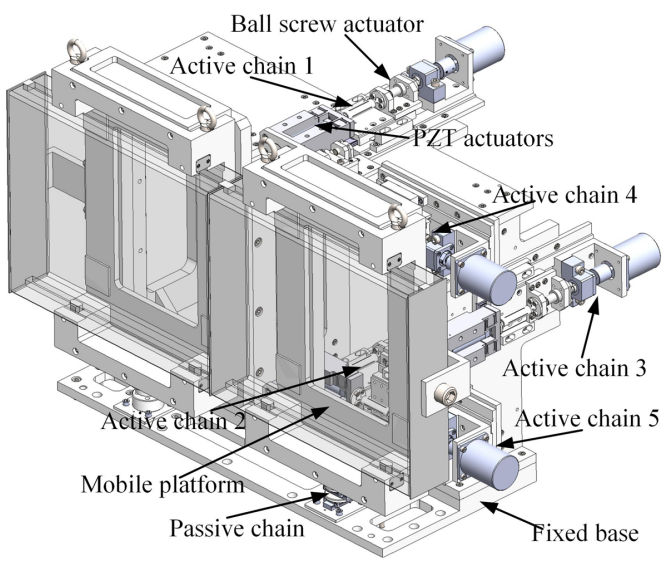

(a)

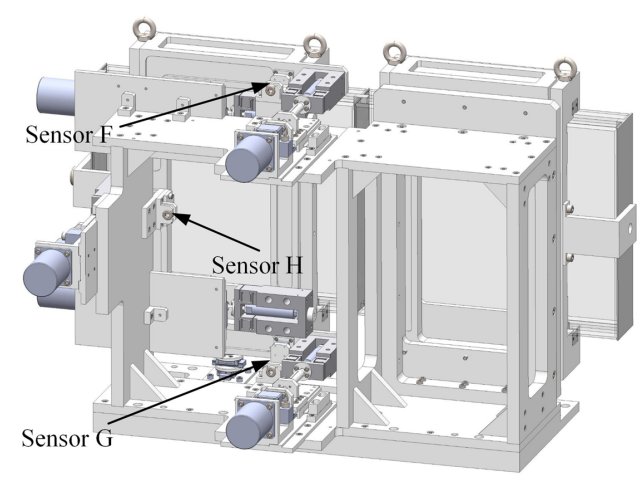

(b)

Figure 2. Structure of grating tiling device. (a) Front view, (b) rear view.

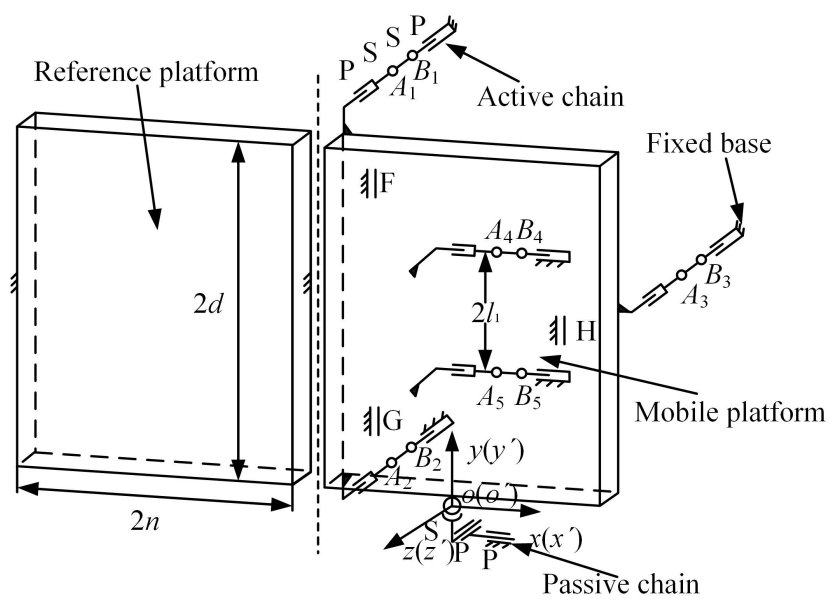

Figure 3. Sketch of grating tiling device.

The spatial 5-PSS-PPS parallel structure combines with the 5-SSP-PPS parallel structure to form a 5-DOF 5PSS/SSP-PPS macro-micro compliant parallel mechanism. The 5-PSS/SSP-PPS mechanism can achieve 5-DOF movements, including 2 translational DOFs along the $x$ and $z$ axes and 3 rotational DOFs about the $x, y$ and $z$ axes. The mechanism is composed of a fixed base, a mobile platform, a properly constrained passive chain and five identical unconstrained active chains with six degrees of freedoms, which connect the mobile platform and the fixed base. The six kinematic chains adopt a three-orthogonal-plane and single-point supporting layout. When the grating tiling device works, the preparatory adjustment is accomplished by the macromotion. The macromotion can be locked after its moving range shifts into the movement range of micromotion. Then, the micromotion is activated. The mobile platform can achieve 5-DOF large-stroke, high-precision and high-stability control.

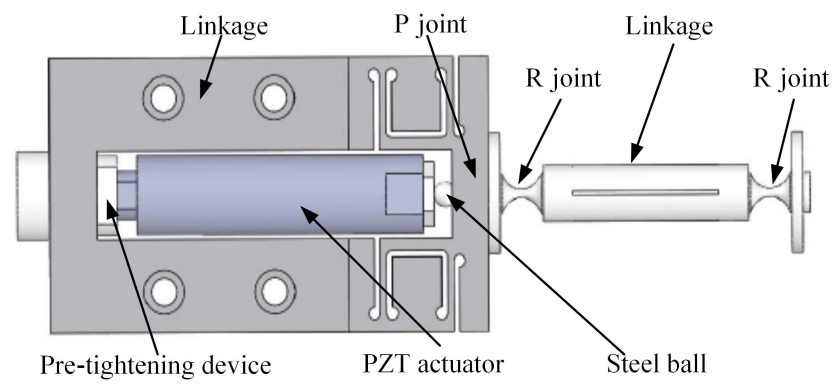

Figure 4. A single compliant chain.

\subsection{Design of flexure hinges}

The structure of unconstrained active chains is illustrated in Fig. 4. Compared with various flexure hinges, the right circular flexure hinge is considered a good candidate because of its excellent rotation capacity and lack of rotation errors (Lobontiu, 2002; Chen et al., 2008, 2009). Hence, the right circle flexure spherical joint as shown in Fig. 5 is adopted in the prototype. The double linear spring parallel combination of two linear springs (Fig. 6) is used as a flexure prismatic joint. This double linear spring not only reduces the coupling error of the single linear springs, but also increases the stiffness of the structure. And the anti-interference ability ensures that the structure has only one degree of freedom in the direction of motion, which can improve the guiding precision and working performance of the system. The PZT actuator is fixed using a pre-tightening device and a steel ball. The pretightening device ensures firm contact and the steel ball can help PZT actuators to withstand shear forces.

The grating tiling mechanism stiffness is determined by the stiffness of the flexure hinge, which plays an important role in the design of a compliant parallel mechanism. To increase the natural frequency of the mechanism so as to improve the stability and dynamic response, the design princi- 


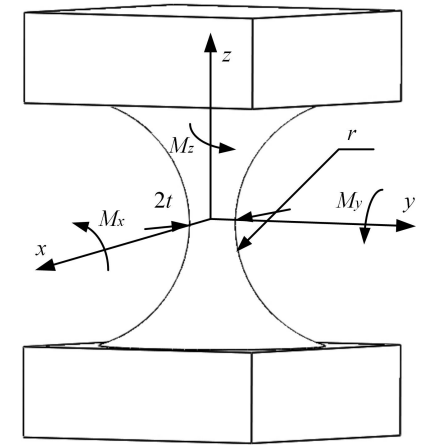

Figure 5. Right circle spherical joint.

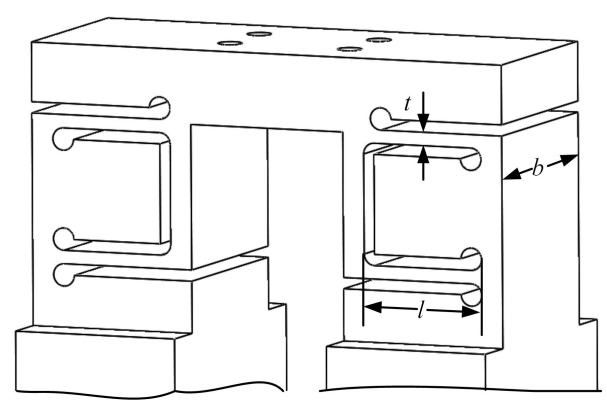

Figure 6. Double linear spring prismatic joint.

ple of the flexure hinge stiffness is that the stiffness should be as large as possible under the constraint of the stroke.

The $\mathrm{S}$ joint can achieve 3 rotational DOFs around three coordinate axes. According to Castigliano's theorem (Lobontiu, 2002), the relationship of deflections $\delta \theta=\left(\theta_{x} \theta_{y} \theta_{z}\right)$ and moments $M=\left(M_{x} M_{y} M_{z}\right)$ applied on the joint can be expressed by

$\delta \theta=C_{\mathrm{R}} M$,

$C_{\mathrm{R}}=\operatorname{diag}\left(C_{x} C_{y} C_{z}\right)$,

$C_{x}=C_{y}=\frac{4 r}{\pi E} \int_{-\frac{\pi}{2}}^{\frac{\pi}{2}} \frac{\cos \theta}{(t+r-r \cos \theta)^{4}} \mathrm{~d} \theta$,

$C_{z}=\frac{2 r}{\pi G} \int_{-\frac{\pi}{2}}^{\frac{\pi}{2}} \frac{\cos \theta}{(t+r-r \cos \theta)^{4}} \mathrm{~d} \theta$,

where $C_{\mathrm{R}}$ is the compliance of the spherical joint, $E$ is Young's modulus and $r$ and $t$ are the geometric parameters of the spherical joint.

Based on the stiffness matrices method (Pham and Chen, 2005), the relationship between the applied force $F_{y}$ and de-

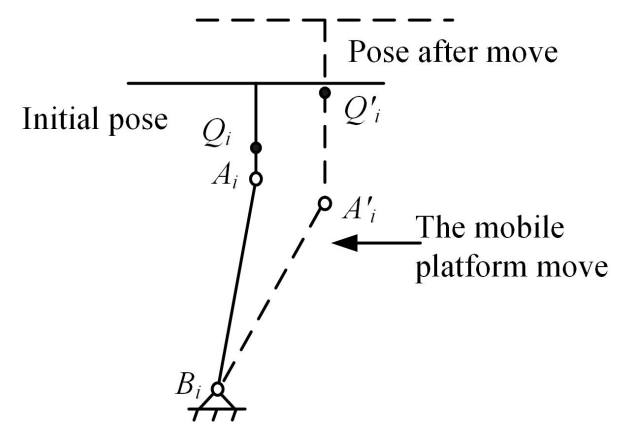

Figure 7. Sketch of micromotion.

formation $\delta_{y}$ can be derived as follows:

$\delta y=C_{\mathrm{P}} F_{y}$,

$C_{\mathrm{P}}=\frac{l^{3}}{4 E b t^{3}}+\frac{l}{4 G b t}$,

where $C_{\mathrm{P}}$ is the compliance of the prismatic joint, $G$ is the shear modulus and $t, b$ and $l$ are the geometric parameters of the prismatic joint.

\section{Kinematics analysis of the 5-DOF grating tiling mechanism}

For kinematics analysis, a fixed coordinate system $o-x y z$ at the center of a steel ball and a local reference coordinate systems $o^{\prime}-x^{\prime} y^{\prime} z^{\prime}$ are assigned, respectively, as is shown in Fig. 3. The coordinate system $o-x y z$ and $o^{\prime}-x^{\prime} y^{\prime} z^{\prime}$ coincide in the initial position. The micromotion has 5 DOFs, so a vector $X=(x z \alpha \beta \gamma)$ is used to describe the motion of the mobile platform. As shown in Fig. 7, the position of point $B_{i}$ on each kinematic chain is fixed and point $A_{i}$ moves to point $A_{i}^{\prime}$ when the platform moves. However, the linkage length between point $A_{i}^{\prime}$ and point $B_{i}$ is constant. According to the above condition, the position solution can be derived

$L^{2}=\left|A_{i} B_{i}\right|^{2}=\left|A_{i}^{\prime} B_{i}\right|^{2}=\left(A_{i}^{\prime}-B_{i}\right)^{T}\left(A_{i}^{\prime}-B_{i}\right), i=1,2 \ldots .$.

where $A_{i}^{\prime}\left(a_{i x}^{\prime}, a_{i y}^{\prime}, a_{i z}^{\prime}\right)$ and $B\left(b_{i x}, b_{i y}, b_{i z}\right)$ are the generalized coordinates of points $A_{i}^{\prime}$ and $B_{i}$ in the fixed coordinate system and $L$ is the linkage length between point $A_{i}^{\prime}$ and point $B_{i}$. The coordinate of $A_{i}^{\prime}$ can be obtained by using

$A_{i}^{\prime}=Q_{i}^{\prime}+d_{i} \cdot i^{\prime}, i=1,2 \ldots 5$,

where $Q_{i}^{\prime}\left(q_{i x}^{\prime}, q_{i y}^{\prime}, q_{i z}^{\prime}\right)$ is the coordinate of point $Q_{i}^{\prime}$ in the fixed coordinate system, which is fixed relative to the mobile platform, $d_{i}$ is the length of $Q_{i}^{\prime} A_{i}^{\prime}$ and $i^{\prime}\left(i_{x}^{\prime}, i_{y}^{\prime}, i_{z}^{\prime}\right)$ is a direction vector of $Q_{i}^{\prime} A_{i}^{\prime}$.

The coordinates of $A_{i}$ also can be calculated by using

$A_{i}^{\prime}=T \cdot Q_{i}+d_{i} \cdot \operatorname{Tr} \cdot \boldsymbol{i}, i=1,2 \ldots .5$, 
$T=\left[\begin{array}{rrrr}1 & -\chi & \beta & x \\ \chi & 1 & -\alpha & 0 \\ -\beta & \alpha & 1 & z \\ 0 & 0 & 0 & 1\end{array}\right]$

$\operatorname{Tr}=\left[\begin{array}{rrrr}1 & -\chi & \beta & 0 \\ \chi & 1 & -\alpha & 0 \\ -\beta & \alpha & 1 & 0 \\ 0 & 0 & 0 & 1\end{array}\right]$,

where $T$ is the homogenous transformation matrix from $o^{\prime}$ $x^{\prime} y^{\prime} z^{\prime}$ to $o-x y z, T r$ is the rotation transformation matrix, $Q_{i}$ is the coordinate of point $Q_{i}$ in the local frame and $i$ is a direction vector of $Q_{i} A_{i}$.

The relationship between $d_{i}$ and the end pose of the mobile platform is obtained with

$$
\begin{aligned}
L^{2} & =\left(q_{i x}^{\prime}+d_{i} \cdot i_{x}^{\prime}-b_{i x}\right)^{2}+\left(q_{i y}^{\prime}+d_{i} \cdot \boldsymbol{i}_{y}^{\prime}-b_{i y}\right)^{2} \\
& +\left(q_{i z}^{\prime}+d_{i} \cdot \boldsymbol{i}_{z}^{\prime}-b_{i z}\right)^{2} .
\end{aligned}
$$

According to Eq. (12), the following relationship can be obtained.

$d_{i}=\frac{-b-\sqrt{b^{2}-4 a c}}{2 a}$

$a=i_{x}^{\prime 2}+i_{y}^{\prime 2}+i_{z}^{\prime 2}$

$b=2\left(q_{i x}^{\prime}-b_{i x}^{\prime}\right)+2\left(q_{i y}^{\prime}-b_{i y}^{\prime}\right)+2\left(q_{i z}^{\prime}-b_{i z}^{\prime}\right)$

$c=\left(q_{i x}^{\prime}-b_{i x}^{\prime}\right)^{2}+\left(q_{i y}^{\prime}-b_{i y}^{\prime}\right)^{2}+\left(q_{i z}^{\prime}-b_{i z}^{\prime}\right)^{2}$

The following equation is then obtained:

$p_{i}=d_{i}-d_{0}$,

where $p_{i}$ is input displacement and $d_{0}$ is the length of $Q_{i} A_{i}$.

Thus, the relationship between the pose of the mobile platform and input displacement can be written as follows:

$\left[\begin{array}{l}p_{1} \\ p_{2} \\ p_{3} \\ p_{4} \\ p_{5}\end{array}\right]=T_{\text {micro }}\left[\begin{array}{c}x \\ z \\ \alpha \\ \beta \\ \gamma\end{array}\right]$

where

$$
T_{\text {micro }}=\left[\begin{array}{ccccc}
\frac{\partial p_{1}}{\partial x} & \frac{\partial p_{1}}{\partial y} & \frac{\partial p_{1}}{\partial \alpha} & \frac{\partial p_{1}}{\partial \beta} & \frac{\partial p_{1}}{\partial \gamma} \\
\frac{\partial p_{2}}{\partial x} & \frac{\partial p_{2}}{\partial y} & \frac{\partial p_{2}}{\partial \alpha} & \frac{\partial p_{2}}{\partial \beta} & \frac{\partial p_{2}}{\partial \gamma} \\
\frac{\partial p_{3}}{\partial x} & \frac{\partial p_{3}}{\partial y} & \frac{\partial p_{3}}{\partial \alpha} & \frac{\partial p_{3}}{\partial \beta} & \frac{\partial p_{3}}{\partial \gamma} \\
\frac{\partial p_{4}}{\partial x} & \frac{\partial p_{4}}{\partial y} & \frac{\partial p_{4}}{\partial \alpha} & \frac{\partial p_{4}}{\partial \beta} & \frac{\partial p_{4}}{\partial \gamma} \\
\frac{\partial p_{5}}{\partial x} & \frac{\partial p_{5}}{\partial y} & \frac{\partial p_{5}}{\partial \alpha} & \frac{\partial p_{5}}{\partial \beta} & \frac{\partial p_{5}}{\partial \gamma}
\end{array}\right] .
$$

The initial value $[0,0,0,0,0]^{T}$ and the mechanism parameters are substituted into Eq. (19), and therefore

$$
\left[\begin{array}{l}
p_{1} \\
p_{2} \\
p_{3} \\
p_{4} \\
p_{5}
\end{array}\right]=\left[\begin{array}{rcccc}
0 & 1 l & 2 d+r_{1} & n & 0 \\
0 & 1 & r_{1} & n & 0 \\
0 & 1 & d+r_{1} & -n & 0 \\
-1 & 0 & 0 & m & d+l_{1}+r_{1} \\
-1 & 0 & 0 & m & d-l_{1}+r_{1}
\end{array}\right]\left[\begin{array}{c}
x \\
z \\
\alpha \\
\beta \\
\gamma
\end{array}\right],
$$

where $2 d$ is the width of the mobile platform, $2 n$ is the length of the mobile platform, $r_{1}$ is the radius of the steel ball, $m$ is the distance between kinematic chains 4 and 5 and the $x$ $y$ plane, and $2 l_{1}$ is the distance between kinematic chains 4 and 5 .

Similarly, the relationship between the pose of the mobile platform and input displacement of macromotion can be written as follows:

$\left[\begin{array}{c}p_{1} \\ p_{2} \\ p_{3} \\ p_{4} \\ p_{5}\end{array}\right]=T_{\text {macro }}\left[\begin{array}{c}x \\ z \\ \alpha \\ \beta \\ \gamma\end{array}\right]$

The transformation matrix $T_{\text {macro }}$ can be expressed as follows

$$
T_{\text {macro }}=\left[\begin{array}{rcccc}
0 & 1 & 2 d+r_{1} & n & 0 \\
0 & 1 & r_{1} & n & 0 \\
0 & 1 & d+r_{1} & -n & 0 \\
-1 & 0 & 0 & m & d+l_{1}+r_{1} \\
-1 & 0 & 0 & m & d-l_{1}+r_{1}
\end{array}\right] .
$$

\section{Full closed-loop control system for stability of grating tiling device}

The vibration in the working environment affects the stability of the grating tiling device. In order to suppress the impact of vibration and improve the stability of the grating tiling device, a full closed-loop control system, which is composed of a kinematics control model, a monitoring model and a PID control model, is designed. The basic flow of the full closedloop control system for the stability of the grating tiling device is as follows. Firstly, the displacement changes of the capacitive position sensors are transformed into the position and angular information of the grating by using the monitoring algorithm. Secondly, it needs to be determined whether it exceeds the threshold. If it exceeds the threshold, the information is transformed into the input displacements of the actuators by using the kinematics control algorithm after it is processed by the PID control algorithm. Finally, the actuators drive the tiling mechanism to the initial position.

\subsection{Monitoring model}

The five degrees of freedom of two adjacent gratings can be divided into three groups and they can compensate for each 


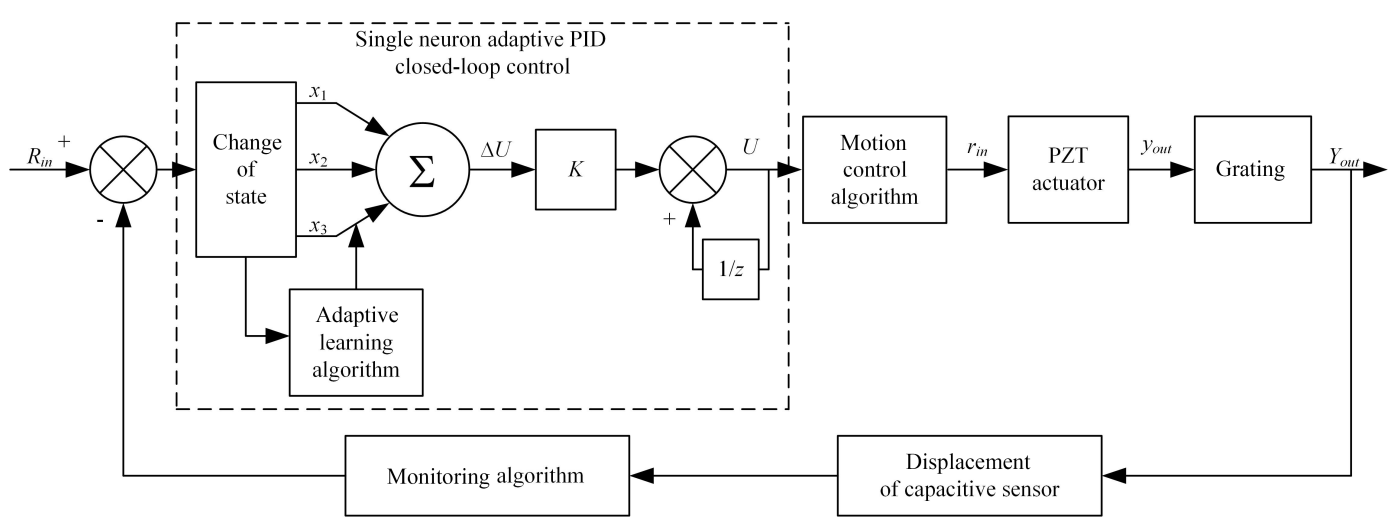

Figure 8. Schematic of single neuron adaptive PID control model.

other according to the compensation pair error theory of grating tiling (Kessler et al., 2004). The three groups are shift linear rotation and angular tip, lateral and longitudinal piston, and angular tilt. Thus, to achieve stability control in terms of only longitudinal piston, angular tip and angular tilt, these 3 degrees of freedom need full closed-loop control.

Capacitive position sensors merely reflect the change in linear displacement. Therefore, it is necessary to study the monitoring algorithm between displacement changes of the capacitive position sensors and the changes in the position and angle of grating tiling. As shown in Fig. 3, three capacitive position sensors $\mathrm{F}, \mathrm{G}$ and $\mathrm{H}$, which are distributed at the vertices of the equilateral triangle and the connection of the capacitive position sensors $\mathrm{F}$ and $\mathrm{G}$ is parallel to the $Y$ axis, are arranged along the $Z$ direction between the mobile platform and the fixed base to sense the actual displacements. The position of the capacitive position sensors can be obtained as follows:

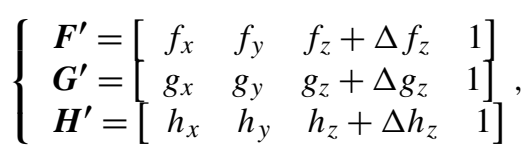

where $\left[\boldsymbol{F}^{\prime}, \boldsymbol{G}^{\prime}, \boldsymbol{H}^{\prime}\right]$ represent the position vectors of the three capacitive position sensors and $\Delta f_{z}, \Delta g_{z}$ and $\Delta h_{z}$ are the feedback displacements.

The relationship between the moving frame $O^{\prime}\left(o^{\prime}-x^{\prime} y^{\prime} z^{\prime}\right)$ unit vector and the position vectors of the three capacitive position sensors can be expressed as

$$
\left\{\begin{aligned}
\boldsymbol{i}_{y^{\prime}} & =\frac{\boldsymbol{G}^{\prime} \boldsymbol{F}^{\prime}}{\left|\boldsymbol{G}^{\prime} \boldsymbol{F}^{\prime}\right|} \\
\boldsymbol{i}_{x^{\prime}} & =i_{y^{\prime}} \times i_{z^{\prime}} \\
\boldsymbol{i}_{z^{\prime}} & =\frac{\boldsymbol{G}^{\prime} \boldsymbol{F}^{\prime} \times \boldsymbol{H}^{\prime} \boldsymbol{F}^{\prime}}{\left|\boldsymbol{G}^{\prime} \boldsymbol{F}^{\prime} \times \boldsymbol{H}^{\prime} \boldsymbol{F}^{\prime}\right|}
\end{aligned}\right.
$$

where $\left[\boldsymbol{i}_{x^{\prime}}, \boldsymbol{i}_{y^{\prime}}, \boldsymbol{i}_{z^{\prime}}\right]$ is a unit vector of the moving frame.

The relative position and the orientation of the moving frame with respect to the fixed reference frame, which can be expressed by Euler angles, are obtained via coordinate conversion as

${ }_{O^{\prime}}^{O} T=\left[\begin{array}{llll}\boldsymbol{i}_{x^{\prime}} \cdot \boldsymbol{i}_{x} & \boldsymbol{i}_{x^{\prime}} \cdot \boldsymbol{i}_{y} & \boldsymbol{i}_{x^{\prime}} \cdot \boldsymbol{i}_{z} & x \\ \boldsymbol{i}_{y^{\prime}} \cdot \boldsymbol{i}_{x} & \boldsymbol{i}_{y^{\prime}} \cdot \boldsymbol{i}_{y} & \boldsymbol{i}_{y^{\prime}} \cdot \boldsymbol{i}_{z} & y \\ \boldsymbol{i}_{z^{\prime}} \cdot \boldsymbol{i}_{x} & \boldsymbol{i}_{z^{\prime}} \cdot \boldsymbol{i}_{y} & \boldsymbol{i}_{z^{\prime}} \cdot \boldsymbol{i}_{z} & z \\ 0 & 0 & 0 & 1\end{array}\right]=T$,

where $\left[\boldsymbol{i}_{x}, \boldsymbol{i}_{y}, \boldsymbol{i}_{z}\right]$ is a unit vector of the fixed reference frame.

Thus, the Euler angles can be expressed as follows:

$$
\left\{\begin{array}{l}
\alpha=\arcsin \left(-\frac{\boldsymbol{i}_{x^{\prime}} \cdot \boldsymbol{i}_{y}}{\cos (\beta)}\right) \\
\beta=\arcsin \left(\boldsymbol{i}_{x^{\prime}} \cdot \boldsymbol{i}_{z}\right) \\
\gamma=\arcsin \left(-\frac{\boldsymbol{i}_{y^{\prime}} \cdot \boldsymbol{i}_{z}}{\cos (\beta)}\right)
\end{array} .\right.
$$

The mobile platform and capacitive position sensors have the same angular displacements but different linear displacements. The displacements of the mobile platform can be expressed as

$\mathbf{P}=\mathbf{J}_{i} \mathbf{X}_{i}$,

where $\mathbf{J}_{i}$ is the transformation matrix from the reference point of the $i$ th capacitive position sensor to the reference point of the fixed frame, and $\mathbf{J}_{i}$ can be derived as follows:

$\mathbf{J}_{i}=\left[\begin{array}{rrrrrr}1 & 0 & 0 & 0 & r_{i z} & -r_{i y} \\ 0 & 1 & 0 & -r_{i z} & 0 & r_{i x} \\ 0 & 0 & 1 & r_{i y} & -r_{i x} & 0 \\ 0 & 0 & 0 & 1 & 0 & 0 \\ 0 & 0 & 0 & 0 & 1 & 0 \\ 0 & 0 & 0 & 0 & 0 & 1\end{array}\right]$,

where $r_{i x}, r_{i y}, r_{i z}$ are the components of the vector from the reference tip of the $i$ th capacitive position sensors and the $i$ th limb to the reference point of the platform.

\subsection{Single neuron adaptive PID control model}

In order to effectively suppress the influence of vibration in the working environment, a single neuron adaptive PID control algorithm with self-adaptive and self-learning ability is 


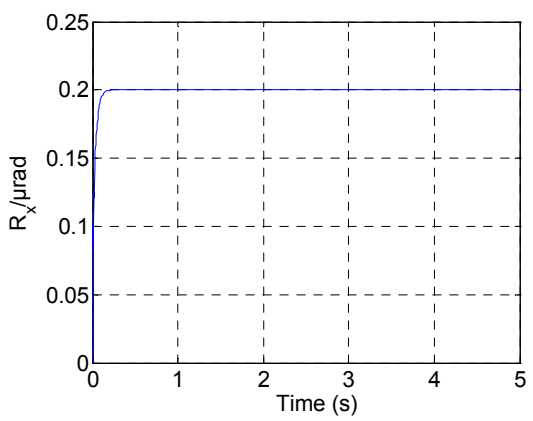

(a)

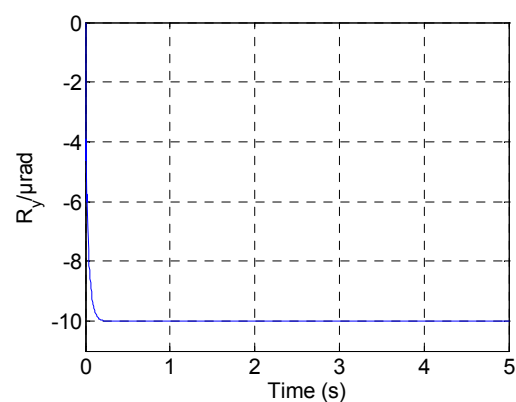

(b)

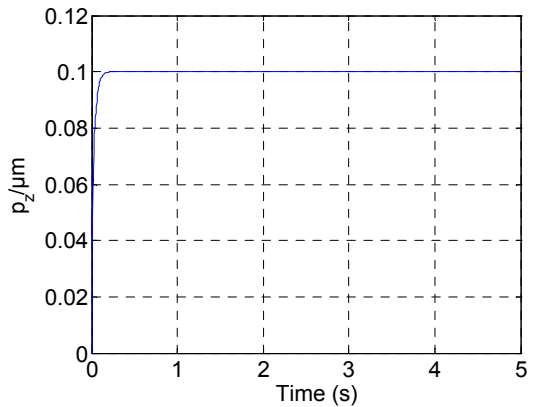

(c)

Figure 9. Out pose simulation results: (a) $R_{x}$, (b) $R_{y}$, (c) $P_{z}$.

applied to realize the full closed-loop control as shown in Fig. 8. Single neuron self-tuning PID control takes the ideal pose $\left(R_{\text {in }}\right)$ as an input and the actual pose as a feedback to achieve the stability of the grating tiling mechanism of the whole closed-loop control.

The single neuron adaptive controller adjusts the weight coefficient according to the supervised Hebb learning rule to realize the adaptive and self-learning function. The control algorithm and learning algorithm can be expressed as fol- lows:

$$
\left\{\begin{aligned}
u(k) & =u(k-1)+K \sum_{i=1}^{3} \omega_{i}(k) x_{i}(k) \\
\omega_{i}^{\prime}(k) & =\omega_{i}(k) / \sum_{i=1}^{3}\left|\omega_{i}(k)\right| \\
\omega_{1}(k) & =\omega_{1}(k-1)+\eta_{\mathrm{I}} z(k) u(k) x_{1}(k) \\
\omega_{2}(k) & =\omega_{2}(k-1)+\eta_{\mathrm{P}} z(k) u(k) x_{2}(k) \\
\omega_{3}(k) & =\omega_{3}(k-1)+\eta_{\mathrm{D}} z(k) u(k) x_{3}(k) \\
x_{1}(k) & =z(k)=e(k)=r(k)-y(k) \\
x_{2}(k) & =e(k)-e(k-1) \\
x_{3}(k) & =e(k)-2 e(k-1)+e(k-2)
\end{aligned}\right.
$$

where, $\eta_{\mathrm{P}}, \eta_{\mathrm{I}}$ and $\eta_{\mathrm{D}}$ are the proportional, integral and differential learning rates, respectively, and $K>0$ is the proportional coefficient of the neuron.

The MATLAB program is combined with ADAMS to simulate the above single neuron PID closed-loop control algorithm. The ideal pose is configured respectively with $R_{x}=$ $0.2 \mu \mathrm{rad}, R_{y}=-10.0 \mu \mathrm{rad}$ and $P_{z}=0.1 \mu \mathrm{m}$. The simulation results are shown in Fig. 9. It can be seen from the figure that the single neuron self-tuning PID full closed-loop control ultimately realized grating pose control with no overshoot, no oscillation and a fast response by configuring reasonable PID control parameters.

\section{Experiment and results}

In order to test the performance of the grating tiling device, experiments for movement accuracy and stability testing are implemented. The grating tiling system requires a nanoscale and micro-arc level of adjustment accuracy, and vibration in the working environment has a great impact on the experimental results. The environment of the experiments requires constant temperature, humidity, quietness and a high level of cleanliness in the laboratory. The experimental system is constructed as shown in Fig. 10. It is mainly composed of a test instrument, a grating tiling prototype and an industrial computer. As the grating is easy damage, experiments are carried out using the same mass of aluminum blocks instead of gratings. In this experiment, the linear motion of the tiling mechanism was tested by using an MT2502 Heidenhain digital length gauge and an ND 280 digital display unit with a resolution of $1 \mathrm{~nm}$. The rotary motion of the tiling mechanism is measured with an Angco Collapex AC300 digital self-collimator with a resolution of 0.01 arcsec.

In this experiment, we measure the motion accuracy of each degree in the negative limiting position, zero point position and positive limiting position by stepping 20 times with a $0.2 \mu \mathrm{rad}$ step rate. From the testing results, it was found that a step accuracy of $20 \mathrm{~nm}$ can be achieved along the $x$ and $z$ axes. The testing results around the $X$ axis, $Y$ axis and $Z$ axis are shown in Tables 1, 2 and 3, respectively. Mathematical statistics are used to perform data processing, and the motion accuracy is evaluated by the average, standard devia- 

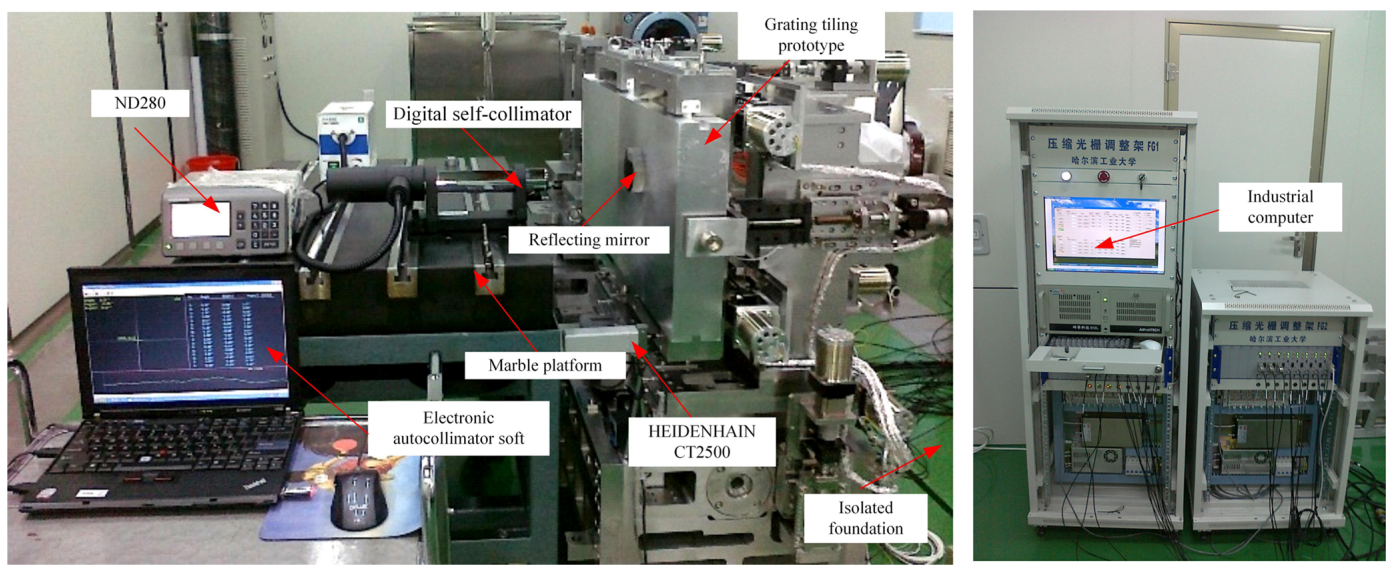

Figure 10. Experimental system.

Table 1. Testing results around the $X$ axis ( $\mu \mathrm{rad})$.

\begin{tabular}{llll}
\hline Position & $\begin{array}{l}\text { Negative } \\
\text { limiting }\end{array}$ & $\begin{array}{l}\text { Zero } \\
\text { point }\end{array}$ & $\begin{array}{l}\text { Positive } \\
\text { limiting }\end{array}$ \\
\hline Average & 0.2088 & 0.2054 & 0.2109 \\
Standard deviation & 0.0462 & 0.0316 & 0.0352 \\
$E$ & $4.4 \%$ & $2.7 \%$ & $5.45 \%$ \\
\hline
\end{tabular}

Table 2. Testing results around the $Y$ axis ( $\mu \mathrm{rad})$.

\begin{tabular}{llll}
\hline Position & $\begin{array}{l}\text { Negative } \\
\text { limiting }\end{array}$ & $\begin{array}{l}\text { Zero } \\
\text { point }\end{array}$ & $\begin{array}{l}\text { Positive } \\
\text { limiting }\end{array}$ \\
\hline Average & 0.2052 & 0.1988 & 0.2041 \\
Standard deviation & 0.0406 & 0.0303 & 0.0341 \\
$E$ & $2.6 \%$ & $0.6 \%$ & $2.05 \%$ \\
\hline
\end{tabular}

tion and average relative errors in the step amount. The average relative errors can be formulated as

$E=\left(\sum(\mid\right.$ input - output $\mid /$ input $\left.) \times 100 \%\right) / 20$.

The tables indicate that the average relative errors are below $5.5 \%$ and average close to the step rate. The standard deviations are relatively small, which demonstrates that the mechanism has good step uniformity. The rotational accuracy around the $Y$ axis is better than that around the $X$ axis and the $Z$ axis. From the statistical results, it can be seen that movement errors are within the scope of control. The main causes for movement errors are as follows. The first cause might be manufacturing and assembly errors, especially for the flexible hinge. Secondly, the resolution of the electronic autocollimator with 0.01 arcsec might have inevitable errors. Last but not least, an experimental environment not at an ideal constant temperature and a vibration isolation environment can also contribute to movement error.
Table 3. Testing results around the $Z$ axis ( $\mu \mathrm{rad})$.

\begin{tabular}{llll}
\hline Position & $\begin{array}{l}\text { Negative } \\
\text { limiting }\end{array}$ & $\begin{array}{l}\text { Zero } \\
\text { point }\end{array}$ & $\begin{array}{l}\text { Positive } \\
\text { limiting }\end{array}$ \\
\hline Average & 0.206 & 0.2089 & 0.210 \\
Standard deviation & 0.0312 & 0.0434 & 0.0431 \\
$E$ & $3.0 \%$ & $4.45 \%$ & $5.0 \%$ \\
\hline
\end{tabular}

Table 4. Stability test results of $\alpha, \beta$ and $z$.

\begin{tabular}{lrrr}
\hline Time & $\alpha\left({ }^{\prime \prime}\right)$ & $\beta\left(^{\prime \prime}\right)$ & $z(\mu \mathrm{m})$ \\
\hline $20: 23: 40$ & 0.04 & -0.04 & 0.050 \\
$20: 53: 40$ & 0.15 & -0.11 & 0.072 \\
$21: 23: 40$ & 0.22 & -0.14 & 0.096 \\
$21: 53: 40$ & 0.20 & -0.39 & 0.131 \\
$22: 23: 40$ & 0.25 & -0.51 & 0.160 \\
\hline
\end{tabular}

To test the stability of the grating tiling prototype under full closed-loop control, longitudinal piston, angular tip and angular tilt, 3-DOF motion tests, which record a set of data every $30 \mathrm{~min}$, are implemented to evaluate the performance of the device. Table 4 shows the stability testing results of 3 degrees of freedom. The maximum change in the 2 rotational DOFs every $30 \mathrm{~min}$ is $1.2 \mu \mathrm{rad}$, while the maximum change in translational DOF is $35 \mathrm{~nm}$.

According to a series of testing results, the grating tiling system can achieve the anticipated accuracy of $0.2 \mu \mathrm{rad}$ in the rotational DOFs. The testing results also indicate that the maximum change in the degree of freedom of rotation is $1.2 \mu \mathrm{rad}$ per $30 \mathrm{~min}$, and the maximum degree of freedom of movement is $35 \mathrm{~nm}$ per $30 \mathrm{~min}$. 


\section{Conclusions}

In this paper, a novel 5-PSS/SSP-PPS compliant parallel mechanism with a three-orthogonal-plane and single-point supporting structure is proposed. The kinematics are derived based on a pseudo-rigid-body model. To achieve high stability, a full closed-loop control system a including monitoring model and a PID control model is proposed. The accuracy and stability testing results show that the step accuracy reaches $0.2 \mu \mathrm{rad}$ per step and $20 \mathrm{~nm}$ per step, and the tiling position retention stability can achieve $1.2 \mu \mathrm{rad}$ per $30 \mathrm{~min}$ and $35 \mathrm{~nm}$ per $30 \mathrm{~min}$ in the rotational direction and the translational direction, which demonstrates that the designed device meets the requirements of high precision and high stability. The design strategy can be extended to other applications requiring high-precision and high-stability manipulation.

Data availability. All the data used in this paper can be obtained upon request from the corresponding author.

Competing interests. The authors declare that they have no conflict of interest.

Acknowledgements. This work is partially supported by the National Natural Science Foundation of China (grant no. 51475114) and the Natural Science Foundation of Hei Long Jiang Province (grant no. E201424).

Edited by: Guangbo Hao

Reviewed by: Shixun Fan and one anonymous referee

\section{References}

Bai, Q., Liang, Y., Cheng, K., and Long, F.: Design and analysis of a novel large-aperture grating device and its experimental validation, P. I. Mech. Eng. B J. Eng., 227, 1349-1359, 2013.

Cai, K., Tian, Y., Wang, F., Zhang, D., Liu, X., and Shirinzadeh, B.: Design and control of a 6-degree-of-freedom precision positioning system, Robot. Com. Int. Manuf., 44, 77-96, 2017.

Chen, G., Shao, X., Huang, X.: A new generalized model for elliptical arc flexure hinges, Rev. Sci. Instrum., 79, 095103, https://doi.org/10.1063/1.2976756, 2008.

Chen, G., Liu, X., Gao, H., and Jia, J.: A generalized model for conic flexure hinges, Rev. Sci. Instrum., 80, 055106, https://doi.org/10.1063/1.3137074, 2009.

Du, Z., Shi, R., and Dong, W.: A Piezo-Actuated High-Precision Flexible Parallel Pointing Mechanism: Conceptual Design, Development, and Experiments, IEEE T. Robot., 30, 131-137, 2014

Habara, H., Xu, G., Jitsuno, T., Kodama, R., Suzuki, K., Sawai, K., Kondo, K., Miyanaga, N., Tanaka, K. A., Mima, K., Rushford, M. C., Britten, J. A., and Barty, C. P.: Pulse compression and beam focusing with segmented diffraction gratings in a high- power chirped-pulse amplification glass laser system, Opt. Lett., 35, 1783-1785, 2010.

Hale, L. C.: Principles and techniques for designing precision machines, Massachusetts Institute of Technology, Massachusetts, 1999.

Hao, G.: Determinate design and analytical analysis of a class of symmetrical flexure guiding mechanisms for linear actuators, J. Mech. Design, 139, 012301-012301-12, 2016.

Hao, G.: Design and analysis of symmetric and compact 2R1T (inplane 3-DOC) flexure parallel mechanisms, Mech. Sci., 8, 1-9, https://doi.org/10.5194/ms-8-1-2017, 2017.

Hao, G. and Kong, X.: A structure design method for compliant parallel manipulators with actuation isolation, Mech. Sci., 7, 247253, https://doi.org/10.5194/ms-7-247-2016, 2016.

Hao, G. and Yu, J.: Design, modelling and analysis of a completelydecoupled XY compliant parallel manipulator, Mech. Mach. Theory, 102, 179-195, 2016.

Hopkins, J. B. and Culpepper, M. L.: Synthesis of multi-degree of freedom, parallel flexure system concepts via Freedom and Constraint Topology (FACT) Part I: Principles, Precis. Eng., 34, 259$270,2010$.

Hornung, M., Bödefeld, R., Siebold, M., Schnepp, M., Hein, J., Sauerbrey, R., and Kaluza, M. C.: Alignment of a tiled-grating compressor in a high-power chirped-pulse amplification laser system, Appl. Optics, 46, 732-735, 2007.

Kessler, T. J., Bunkenburg, J., Huang, H., Kozlov, A., and Meyerhofer, D. D.: Demonstration of coherent addition of multiple gratings for high-energy chirped-pulse-amplified lasers, Opt. Lett., 29, 635, 2004.

Liang, Q., Zhang, D., Chi, Z., Song, Q., Ge, Y., and Ge, Y.: Six-DOF micro-manipulator based on compliant parallel mechanism with integrated force sensor, Robot. Com. Int. Manuf., 27, 124-134, 2011.

Lobontiu, N.: Design of circular cross-section corner-filleted flexure hinges for three-dimensional compliant, J. Mech. Design, 124, 479-484, 2002.

Pham, H. H. and Chen, I. M.: Stiffness modeling of flexure parallel mechanism, Precis. Eng., 29, 467-478, 2005.

Qiao, J., Kalb, A., Guardalben, M. J., King, G., Canning, D., and Kelly, J. H.: Large-aperture grating tiling by interferometry for petawatt chirped pulse amplification systems, Opt. Express, 15, 9562-9574, 2007.

Shi, H. and Su, H. J.: An Analytical Model for Calculating the Workspace of a Flexure Hexapod Nanopositioner, J. Mech. Robot., 5, 041009-041009, 2013.

Shi, H., Su, H. J., Dagalakis, N., and Kramar, J. A.: Kinematic modeling and calibration of a flexure based hexapod nanopositioner, Precis. Eng., 37, 117-128, 2013.

Shi, H., Su, H. J., and Dagalakis, N.: A stiffness model for control and analysis of a MEMS hexapod nanopositioner, Mech. Mach. Theory, 80, 246-264, 2014.

Su, H. J., Dorozhkin, D. V., and Vance, J. M.: A Screw Theory Approach for the Conceptual Design of Flexible Joints for Compliant Mechanisms, J. Mech. Robot., 1, 41009-41001, 2009.

Yang, Y., Wang, X., Zhang, J., Luo, H., Li, F., Huang, X., and Jing, F.: Automatic phase-locked control of grating tiling, Opt. Laser Eng., 50, 262-267, 2012.

Yu, J., Xie, Y., Li, Z., and Hao, G.: Design and Experimental Testing of an Improved Large-Range Decoupled XY Com- 
pliant Parallel Micromanipulator, J. Mech. Robot., 7, 044503, https://doi.org/10.1115/1.4030467, 2015.

Yun, Y. and Li, Y.: Design and analysis of a novel 6-DOF redundant actuated parallel robot with compliant hinges for high precision positioning, Nonlin. Dynam., 61, 829-845, 2010.
Zhang, T., Yonemura, M., and Kato, Y.: An array-grating compressor for high-power chirped-pulse amplification lasers, Opt. Commun., 145, 367-376, 1998. 